\title{
Multivariate Analysis of Neisseria DNA Restriction Endonuclease Patterns
}

\author{
By B. SøRENSEN,${ }^{*} \dagger$ E. S. FALK,${ }^{2}$ E. WISL $\varnothing F F-N I L S E N,{ }^{3}$ \\ B. BJORVATN ${ }^{4}$ AND B. E. KRISTIANSEN ${ }^{1}$ \\ ${ }^{1}$ Department of Microbiology and ${ }^{2}$ Department of Dermatology, University Hospital of Tromsø, \\ $N$-9012 Tromso, Norway \\ ${ }^{3}$ Department of Chemistry, Institute of Mathematical and Physical Science, University of Tromsø, \\ $N-9000$ Tromso, Norway \\ ${ }^{4}$ Department of Microbiology, Ulleval Hospital, Oslo, Norway
}

(Received 8 January 1985; revised 28 June 1985)

Chromosomal DNA was extracted from eleven Neisseria meningitidis and seven Neisseria gonorrhoeae isolates and cleaved with the restriction enzyme $H$ indIII. The DNA fragments were separated according to their size, using a $4 \%$ polyacrylamide gel. The band patterns obtained were digitized and statistically analysed by the SIMCA method. To develop the models for $N$. meningitidis (class 1 ) and $N$. gonorrhoeae (class 2), all eleven meningococci and seven gonococci were used. All strains were classified correctly and showed an extremely good class separation.

\section{INTRODUCTION}

Fingerprinting of chromosomal DNA from bacteria is a recently developed method, which has been used for identifying different clones of Neisseria meningitidis (Bjorvatn et al., 1984; Kristiansen et al., 1984a), and in epidemics to trace an infecting source (Kristiansen et al., $1984 \mathrm{~b}$ ). The fingerprints have until now been analysed by visual comparison. This has been convenient for comparison of different isolates where the aim has been to detect a complete, or almost complete, identical band pattern from different bacterial strains. To use a visual comparison of the DNA band patterns as a basis for a classification of bacterial strains into genetically similar classes is not satisfactory. An analysis of one variable (here a band) at a time cannot reveal the multivariate information in the complete fingerprint. In addition a more serious problem is the evaluation of the data. Consider, for example, two different classes of strains with say $\mathbf{P}$ different bands. The risk that a difference between two class averages of a single band is due to chance will be $1-(0.95)^{\mathrm{P}} \%$. With $\mathrm{P}=50$ the probability will be about $90 \%$ (Wold et al., 1983). The band pattern should therefore be regarded as a multivariate data set where the analysis is done simultaneously on all bands.

In this paper we report the preparation of the data set for each object and the use of DNA fingerprints analysed by the SIMCA (soft independent modelling of class analogy) method for multivariate pattern recognition as a basis for classification of bacterial strains.

\section{METHODS}

Bacteria. Eleven strains of serogroup B and serotype 15 (B15) meningococci (1-11), and seven strains of gonococci (12-18) were included in the study. All strains had been recently isolated from patients in north Norway. Two classes were defined: class 1 consisted of the meningococci and class 2 of the gonococci.

\footnotetext{
† Present address: Telemark Medical Microbiology Laboratory, Postboks 1868, N-3701 Skien, Norway.

Abbreviations: PC, principal component; RSD, residual standard deviation; SIMCA, soft independent modelling of class analogy.
} 
DNA fingerprinting. A detailed procedure for this method is given elsewhere (Bjorvatn et al., 1984). The DNA fingerprinting technique includes the following steps: culture and lysis of the bacteria, extraction of the DNA followed by its cleavage with the appropriate restriction endonuclease (HindIII; Bio-Lab), PAGE of the DNA fragments and finally staining and photography of the band patterns in the gel.

Preparation of the data for SIMCA. The band patterns of each strain on the photo-negative were digitized using a Joyce Loebel Scandig 3 microdensitometer with a resolution of $0.1 \mathrm{~mm}$. The data were then transferred to a Cyber 171 multi-processor where the rest of the work was done.

Two scaling procedures were used. (1) Adjustment of the band patterns on the photo-negative to a common fragment size scale. Escherichia coli phage P4 DNA digested by HindIII was used as a standard. This scaling was necessary because it is difficult to obtain exactly the same size of the different gels after staining and washing procedures. (2) Adjustment of the peak heights representing the band intensity of an internal standard. The band chosen as the internal standard was present in all 18 fingerprints, and had a fragment size of approximately $1.4 \mathrm{~kb}$. The background was chosen to be the lowest pixel on the actual scan plus $10 \%$ of the intensity of the internal standard.

To improve the reproducibility and reduce the amount of variables a numeric 'filter' equivalent to a moving average was used. The filter is described by equation (1), and incorporates small and badly resolved shoulders in the main peaks. In addition it preserves most of the information in the data set and, to a large extent, eliminates false pixels which otherwise may cause problems during the analysis.

$$
V(j+k)=\sum_{i=j}^{j+N} V_{i} / N
$$

where $k=(N+1) / 2, j=$ a variable index, $V_{i}=$ value of variable $i$, and $N=3,5,7,9 \ldots$; in our case $N=9$.

The recorded data set contained about 1000 absorbance measurements. After scaling and filtering, only the DNA fragments between $3.2 \mathrm{~kb}$ and $1.1 \mathrm{~kb}$ were used for the analysis. The number of measurements which describe the DNA fragment patterns within this range is approximately 500. Two examples of different band patterns are shown in Fig. 1. The first scaling procedure causes the distances between each measurement to vary. It was therefore necessary to set up new equally spaced measurements based on interpolation between the original scaled and filtered measurements. We have found that the important information in the band patterns is not lost even if the number of variables is reduced to one-fifth of the original. Hence we have used 100 variables to describe the band pattern of each bacterial strain. These variables are every fifth measurement between $3.2 \mathrm{~kb}$ and $1 \cdot 1 \mathrm{~kb}$.

Principal component $(P C)$ analysis. A PC analysis was made on the whole data set (18 objects with 100 variables). This analysis was used to overview the whole data set without direct reference to class separation.

SIMCA analysis. A detailed description of the principles in disjoint PC modelling (SIMCA) has previously been published (Wold, 1976; Blomquist et al., 1979). In summary, the basic principle of the SIMCA method is to model the data of a class of objects (here $N$. meningitidis or $N$. gonorrhoeae) separately with a PC model. With $X_{i k}(q)$ denoting the measured value of variable $i$ for object $k$ in the class $q$, the class model of class $q$ is a PC model with $A_{q}$ components. If $A_{q}=0$ the model is a point in a space with a dimension equal to the number of variables $(M)$, a line for $A_{q}=1$, a plane for $A_{q}=2$, and so on. The number of significant components for each class model is determined by cross-validation (Wold, 1978).

$$
X_{i k}(q)=a_{i}(q)+\sum_{a=1}^{A q} b_{i a}(q) * t_{a k}(q)+e_{i k}(q)
$$

The SIMCA method has the advantage that it is not restricted to a certain ratio between the number of objects $(N)$ and the number of variables $(M)$. In addition it gives a graphical display of the results which makes their interpretation more convenient. The PC plots of the data are equivalent to eigenvector plots (Kowalski \& Bender, 1973). The projections preserve as much of the data variance in the $M$-space as possible. This means, for example, that objects which tend to lie far away from each other in the $M$-space also tend to lie far away from each other in the projection.

The parameters $a_{i}(q), b_{i a}(q)$ and $t_{a k}(q)$ for $a=1,2 \ldots$, and $A_{q}$ are determined from a least square fit of the class model with $A_{q}$ components to the class training set. The residuals $e_{i k}(q)$ describe the 'errors' in $X_{i k}(q)$ which cannot be described by the class model. The residuals $e_{i k}$ have the residual standard deviation (RSD) $S_{o}$.

$$
S_{o}(q)^{2}=\sum_{i=1}^{M} \sum_{k=1}^{n_{q}} e_{i k}(q)^{2} /\left(M-A_{q}\right)\left(n_{q}-A_{q}-1\right)
$$

where $n_{q}$ is the number of objects in class $q$.

The RSD $S_{o}$ is a 'typical' distance between an object in the class $q$ and the class model. An object $p$ can be classified according to the degree of fit to two or more class models. This fit is accomplished by a multiple 


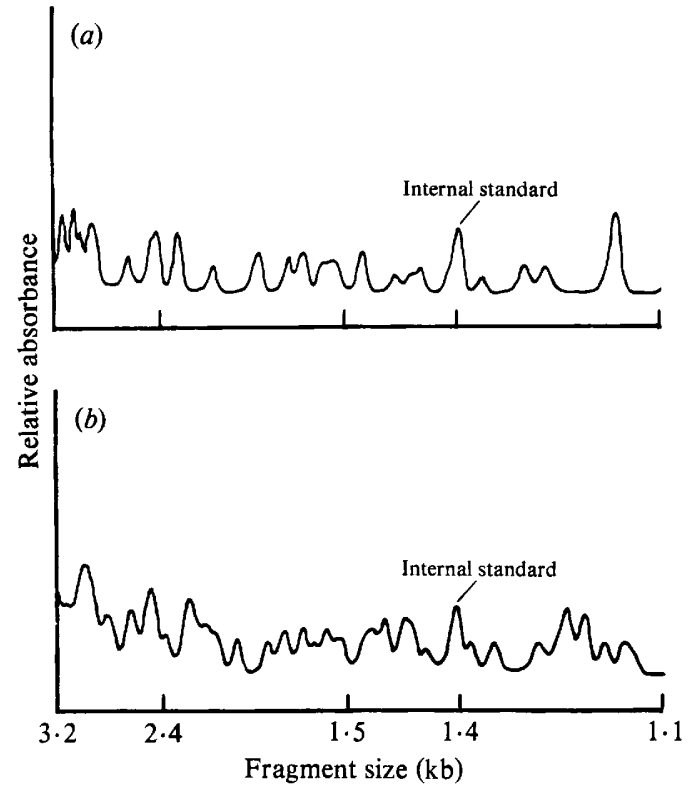

Fig. 1. Typical optical density curves (a) from $N$. gonorrhoeae and $(b)$ from $N$. meningitidis.

regression with $Z_{i}=X_{i}(p)-a_{i}(q)$ as the dependent variable $(i=1,2 \ldots, M)$ and the $A_{q}, b(q)$ vectors as the predictor variables.

$$
Z_{i}(q)=\sum_{a=1}^{A q} b_{i a}(q) * u_{a}(q)+e_{i}(q)
$$

The RSD $S_{p}(q)$ with $\left(M-A_{q}\right)$ degrees of freedom is proportional to the distance between the class model $q$ and the object $p$ in $M$-space.

$$
S_{p}(q)^{2}=\sum_{a=1}^{M} e_{i}(q)^{2} /\left(M-A_{q}\right)
$$

When two class models ( $q$ and $r$ ) are fitted to the data $X_{i}(p)$, the object $p$ is considered to be most similar to the class giving the smaller RSD, $S_{p}(q)$ or $S_{p}(r)$. If, in addition, the smaller RSD is within $\mathrm{F}^{\frac{1}{2} *} S_{o}$ (see equation 5), the object is so similar to the class that it is assigned to it as a member. Hence a SIMCA classification of an object $p$ can result in three different situations: that the object is a member of no class (an outlier), of one class (a unique classification) or of several classes (an ambiguous classification).

The RSD can also be used to detect outliers in class $q$. Thus a training set object $p$ with an $\operatorname{RSD} S_{p}(q)$ greater than the tolerance interval $\left(S_{t o l}\right)$, defined by an approximate F-test with $\left(M-A_{q}\right)$ and $\left(M-A_{q}\right)\left(n_{q}-A_{q}-1\right) / 2$ degrees of freedom, will be defined as an outlier.

$$
S_{t o l}(q)=\mathrm{F}^{\frac{1}{2}} * S_{o}(q)
$$

The top and bottom of the class tolerance intervals are determined on the basis of the distribution of the objects along the $t$-axes, using the sum of $t_{\max }$ and $S_{t}$ as the top and the difference between $t_{\min }$ and $S_{t}$ as the bottom.

$$
S_{t q}(a)=\sum_{k=1}^{n_{q}} t_{a k}(q) / n_{q}
$$

The relevance of the variables can be expressed by their modelling power $\psi$.

$$
\psi_{i}=1-S_{i q} / S_{i q}(x)
$$

Here $S_{i}$ is the RSD of variable $i$ for the class model, and $S_{i}(x)$ is the corresponding RSD of the data $X$ of the class training set.

The object validation for class 1 was made by excluding two objects (strains) from the class training set, and computing a model for the rest of the objects. The two excluded objects were then fitted to the new class model. 
This procedure was done five times successively, going through all the objects belonging to the class training set. The last object was fitted to a model consisting of ten objects instead of nine. For class 2 the object validation was estimated equivalently, but only one object was excluded at a time due to the smaller number of objects in the class.

\section{RESULTS}

Preparation of the variables. The reproducibility of the standardized fingerprint was studied by separating the digested DNA from a single strain on different gels. The localization of the bands between $1.1 \mathrm{~kb}$ and $3.2 \mathrm{~kb}$ fluctuated $0.3 \mathrm{~mm}$ or less after scaling. Bands outside this area were, however, more unstable and difficult to reproduce in this type of gel.

$P C$ analysis. The first four PCs were computed followed by plotting the scores $(t)$ for the first and the second PC. This plot, denoted a PC12 plot, showed a well defined separation between the two classes of bacterial strains (Fig. 2).

SIMCA analysis. Separate models were developed for classes 1 and 2, for both of which the number of components $\left(A_{q}\right)$ was 1 . All 18 objects in the data set were fitted to the models of classes 1 and 2. Fig. 3 shows the separation of the classes using unweighted RSD. The distance between the objects of the two classes that were closest to each other was 13.62 (objects 8 and 12). The model based on class SD for $N$. meningitidis gave three objects lying on the tolerance interval limit defined by the approximate F-test, therefore the interval for the class was extended by $20 \%$. All $N$. gonorrhoeae strains occurred within the $95 \%$ probability level using class SD.

From the object validation, Fig. 3 shows that when object SDs are used the $95 \%$ probability level classifies all objects correctly, and gives an extremely good class separation.

A measure of the relevance of the variables is the modelling power of the PCs. The large number of variables was most easily analysed when the modelling power of the first PC of class 1 was plotted against the modelling power of the first PC of class 2 (Fig. 4). From this plot it is seen

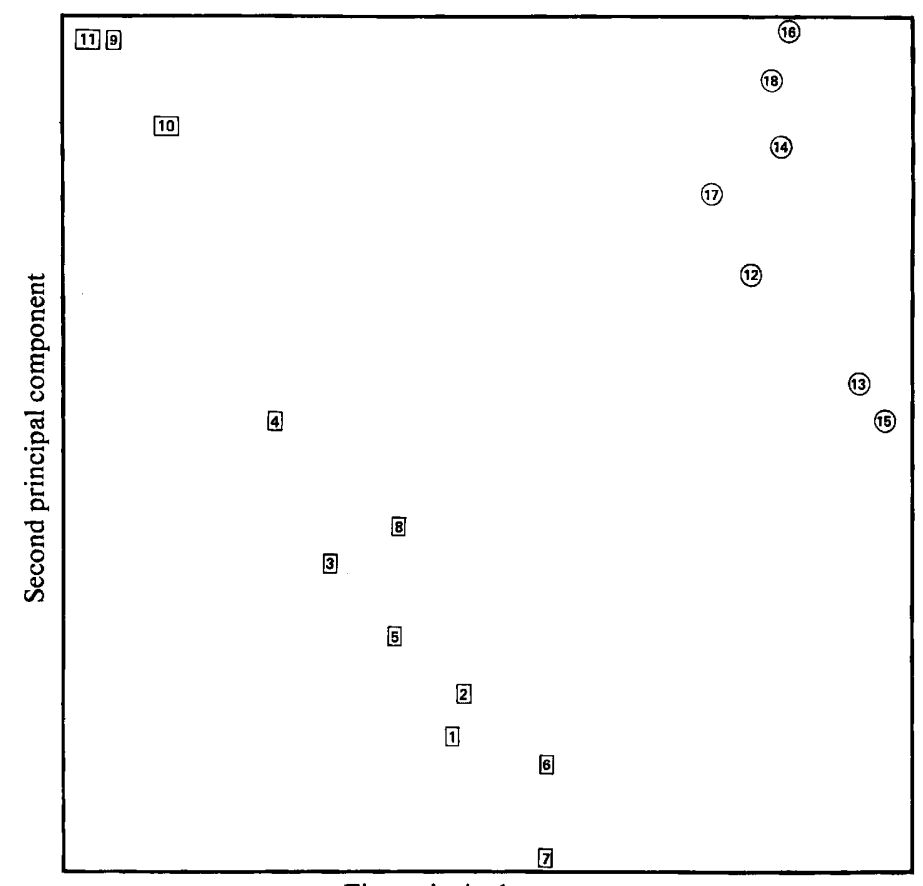

First principal component

Fig. 2. First and second PCs plotted after PC analysis of the whole data set. The numbers refer to the object number in the data set. Squares refer to $N$. meningitidis and circles to $N$. gonorrhoeae. 


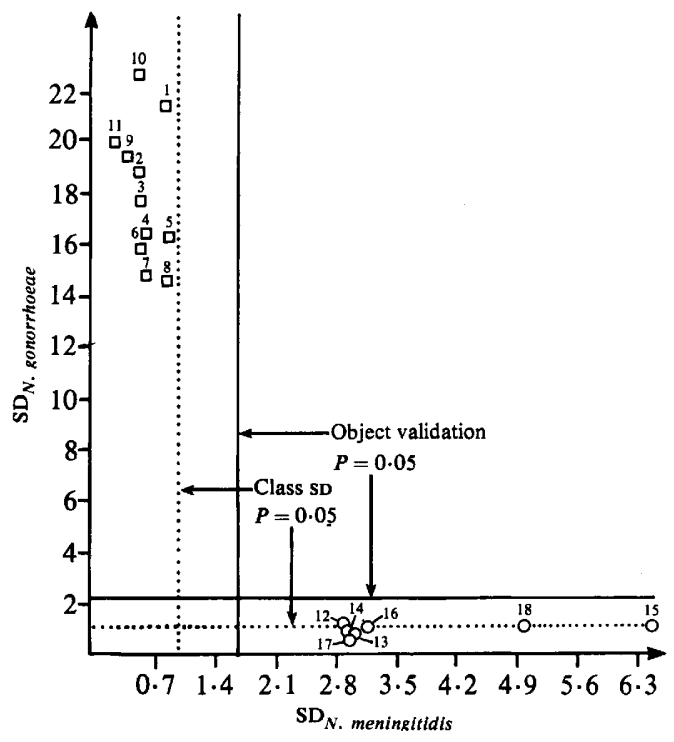

Fig. 3. Class distance plot of $N$. meningitidis and $N$. gonorrhoeae. The DNA fingerprint from each strain was fitted to the two class models; the abscissa represents the SD value obtained when they were fitted to the $N$. gonorrhoeae class model and the ordinate represents the SD values when fitted to the $N$. meningitidis class model. The tolerance interval limits for the two classes are given. Squares refer to $N$. meningitidis and circles to $N$. gonorrhoeae. The numbers refer to the object number in the data set.

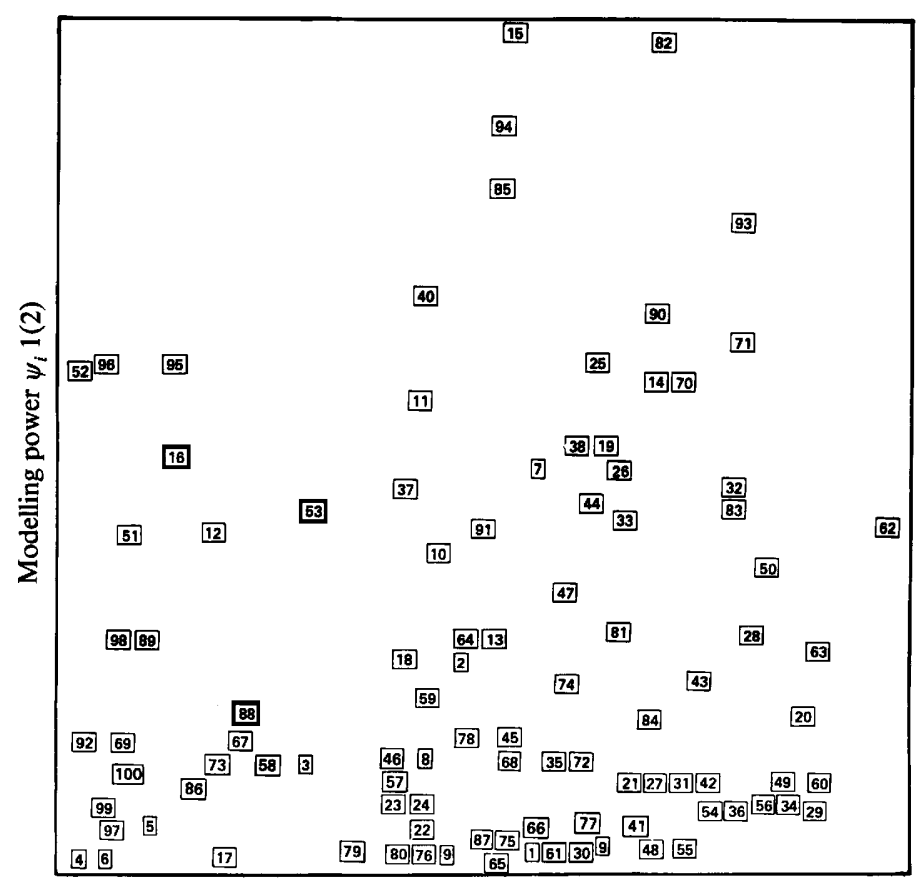

Modelling power $\psi_{i} 1(1)$

Fig. 4. Plot of the modelling power of the different variables (i) for the first PC of class $1\left[\psi_{i} 1(1)\right]$ and class $2\left[\psi_{i} 1(2)\right]$. 
that a dominant part of the variables has high modelling power for $N$. meningitidis, and a relatively minor part of the variables seems to have high modelling power for both $N$. meningitidis and $N$. gonorrhoeae.

\section{DISCUSSION}

The aim of the present study was to establish a method which could transform restriction endonuclease-generated DNA fingerprints into numeric values in such a way that the SIMCA method of data analysis could be used. This would allow measurement of the differences between the DNA fingerprints, thus enabling differentiation of bacterial strains into taxa as well as identification of individual strains within taxa. The value of such a method in epidemiology of bacterial diseases is great.

In a quantitative classification such as this, it is necessary to use an analytical method for the data which can take advantage of the information contained in a multitude of variables even when the number of objects is small. The projection methods such as SIMCA have this property in contrast to traditional methods like linear discriminant analysis (see e.g. Wold et al., 1983).

The number of objects used to build the model for the meningococci was 11 . The object validation of class 1 showed that if the number of objects had been larger it might have been possible to look for subgroups. The model for class 2 was based on seven objects; these objects did form a well defined class, but as seen from Fig. 3, object 15 and object 18 might possibly have formed a subgroup if the number of gonococci had been larger.

A study of the possible correlation between different DNA fragments and, for example, resistance to a distinct antibiotic can be done. The SIMCA method is extendable to analysis of these data by the use of partial least square models instead of PC models (Wold et al., 1983). Preliminary studies in our laboratory indicate such a correlation.

The authors thank Professor S. Wold at the University of Umeå, Sweden, for his constructive criticism of this manuscript

\section{REFERENCES}

Bjorvatn, B., Lund, V., Kristiansen, B. E., Korsnes, L., SPanne, O. \& Lindquist, B. (1984). Applications of restriction endonuclease fingerprinting of chromosomal DNA of Neisseria meningitidis. Journal of Clinical Microbiology 19, 763-765.

Blomquist, G., Johanson, E., SøDerstrøm, B. \& Wold, S. (1979). Classification of fungi by means of pyrolysis-gas chromatography-pattern recognition. Journal of Chromatography 173, 19-32.

Kowalski, B. R. \& Bender, C. F. (1973). Pattern recognition. II. Linear and nonlinear methods for displaying chemical data. Journal of the American Chemical Society 95, 686-693.

Kristiansen, B. E., Sørensen, B., Simonsen, T., Spanne, O., Lund, V. \& BJorvatn, B. (1984a). Isolates of Neisseria meningitidis from different sites on the same patient: phenotypic and genomic studies with special reference to adherence, piliation and DNA restriction endonuclease pattern. Journal of Infectious Diseases 150, 389-396.
Kristiansen, B. E., Sørensen, B., Spanne, O. \& BJORVATN, B. (1984b). Restriction fingerprinting and serology in a small outbreak of B 15 meningococcal disease among Norwegian soldiers. Scandinacian Journal of Infectious Diseases 17, 19-24.

WoLd, S. (1976). Pattern recognition by means of disjoint principal components models. Pattern Recognition 8, 127-139.

Wold, S. (1978). Cross-validatory estimation of the number of components in factor and principal components models. Technometrics 20, 397-405.

Wold, S., Albano, C., Dunn, W. J., III, Esbensen, K., Hellberg, S., Johanson, E. \& Sjøstrøm, M. (1983). Pattern recognition: finding and using regularities in multivariate data. In Food Research and Data Analysis, pp. 147-185. Edited by $\mathrm{H}$. Martens \& H. Russwurm. London \& New York: Applied Science Publishers. 